DRAFT VERSION SEPTEMBER 1, 2016

Preprint typeset using LTEX style emulateapj v. 5/2/11

\title{
TRACING THE REIONIZATION EPOCH WITH ALMA: [CII] EMISSION IN Z 7 GALAXIES
}

\author{
L. Pentericci ${ }^{1}$, S.CARniani ${ }^{2,3}$, M. Castellano ${ }^{1}$, A. Fontana ${ }^{1}$, R. Maiolino ${ }^{2,3}$, L. Guaita $^{1}$, E. VAnZella $^{4}$, A. Grazian $^{1}$, P $^{2}$

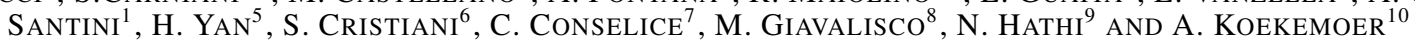 \\ Draft version September 1, 2016
}

\begin{abstract}
We present new results on [CII] $158 \mu \mathrm{m}$ emission from four galaxies in the reionization epoch. These galaxies were previously confirmed to be at redshifts between 6.6 and 7.15 from the presence of the Ly $\alpha$ emission line in their spectra. The Ly $\alpha$ emission line is redshifted by $100-200 \mathrm{~km} \mathrm{~s}^{-1}$ compared to the systemic redshift given by the [CII] line. These velocity offsets are smaller than what is observed in $z \sim 3$ Lyman break galaxies with similar UV luminosities and emission line properties. Smaller velocity shifts reduce the visibility of Ly $\alpha$ and hence somewhat alleviate the need for a very neutral IGM at $z \sim 7$ to explain the drop in the fraction of Ly $\alpha$ emitters observed at this epoch. The galaxies show [CII] emission with $\mathrm{L}[\mathrm{CII}]=0.6-1.6 \times 10^{8} L_{\odot}$ : these luminosities place them consistently below the SFR-L[CII] relation observed for low redshift star forming and metal poor galaxies and also below $\mathrm{z}=5.5$ Lyman break galaxies with similar star formation rates. We argue that previous undetections of [CII] in $z \sim 7$ galaxies with similar or smaller star formation rates are due to selection effects: previous targets were mostly strong $\operatorname{Ly} \alpha$ emitters and therefore probably metal poor systems, while our galaxies are more representative of the general high redshift star forming population.

Subject headings: galaxies: evolution — galaxies: high-redshift — galaxies: formation
\end{abstract}

\section{INTRODUCTION}

We have just entered an exciting era when cosmic microwave background observations can be directly compared to observations of the first galaxies. Reionization is thought to begin at $z \sim 10-15$ and be completed by $z \sim 6$ (Robertson et al. 2015), but how exactly it proceeded in time is still the subject of debate. Understanding the nature of the sources responsible for such process is also an outstanding problem of modern cosmology: both faint galaxies (e.g. Finkelstein et al. 2015) and AGNs (Giallongo et al. 2015) can potentially contribute to reionization and the exact role of the two populations is still unclear.

Deep multi-band imaging surveys identified a large number of candidate galaxies at $z>6$ and up to $z \sim 10$ primarily using the Lyman Break technique but their spectroscopic confirmation has been difficult. At present very few galaxies at $z>7$ are confirmed (e.g. Finkelstein et al. 2013, Oesch et al. 2015, Zitrin et al. 2015). Indeed the extreme difficulty in securing the redshifts of $\mathrm{z}$-dropouts is the first (and perhaps most solid) evidence that the reionization of the Uni-

${ }^{1}$ INAF, Osservatorio Astronomico di Roma, via Frascati 33, 00040 Monteporzio, Italy

${ }^{2}$ Kavli Institute for Cosmology, University of Cambridge, Madingley Road, Cambridge CB3 0HA, UK

${ }^{3}$ Cavendish Laboratory, University of Cambridge, 19 J. J. Thomson Ave., Cambridge CB3 OHE, UK

${ }^{4}$ INAF, Osservatorio Astronomico di Bologna, via Ranzani 1, I-40127 Bologna, Italy

${ }^{5}$ Department of Physics and Astronomy, University of Missouri, Columbia, MO, USA

${ }^{6}$ INAF, Osservatorio Astronomico di Trieste, Via G. B. Tiepolo, 11, I-34143 Trieste, Italy

${ }^{7}$ University of Nottingham, School of Physics and Astronomy, Nottingham NG7 2RD, UK

${ }^{8}$ Astronomy Department, University of Massachusetts, Amherst, MA 01003, USA 0000-0002-7831-8751

9 Aix Marseille UniversitÂl', CNRS, LAM (Laboratoire d'Astrophysique de Marseille

${ }^{10}$ Space Telescope Science Institute, 3700 San Martin Drive, Baltimore, MD 21208, USA verse was not yet complete at $z \simeq 7$. This marked decrease of Ly $\alpha$ emission at $z \sim 7$ compared to $z \sim 6$ is best explained by an increased opacity of the intergalactic medium (IGM) with neutral hydrogen fraction change between the two epochs of $\Delta \chi \sim 0.5$ (Pentericci et al. 2014, Schenker et al. 2014). The physical properties of these galaxies are also uncertain. Star formation rates (SFR) based on UV-luminosity give values of $\sim 10-15 M_{\odot} \mathrm{yr}^{-1}$. However dust is surely present even if in small amounts, at such early epochs (Watson et al. 2015) and can strongly suppress the UV-continuum. Accounting for this may easily raise the inferred SFR to several tens $M_{\odot} y r^{-1}$.

ALMA can play a key role in settling the above issues and assess the nature of high redshift galaxies. The [CII] $157.74 \mu \mathrm{m}$ line, the strongest FIR emission lines in star forming galaxies (accounting for $0.1-1 \%$ of their bolometric luminosity), is accessible by ALMA even at the highest redshifts probed to date. [CII] can provide SFR estimates that are not biased by dust extinction, although they might depend on the metallicity. In addition this line allows us to accurately measure the systemic redshift of the galaxies. The recent [CII] line detections of Lyman Break galaxies (LBGs) and Ly $\alpha$ emitters (LAEs) at $z \sim 5.5-6$ (Capak et al. 2015, Willot et al. 2015) show that the line properties at such high redshifts are similar to those at lower redshift and that they follow a comparable SFR-L[CII] relation (de Looze et al. 2014). The observed galaxies represent the bright end of the UV luminosity function. On the fainter end, Knudsen et al. (2016) detected $[\mathrm{CII}]$ in the strongly lensed $z=6$ galaxy A383-5.1. At variance with the above picture, the few observations attempted on $z \sim 7$ galaxies with ALMA provided contradictory results (e.g. Ota et al. 2012, Schaerer et al. 2014, Maiolino et al. 2015, Watson et al. 2015): several non-detections of [CII] suggested that $z \sim 7$ galaxies have [CII] emission significantly below the expectations from lower redshift relations, although the small number of sources observed and the shallow limits reached for some of them give an uncertain scenario. We previously reported of [CII] observations on three $z \sim 7$ galaxies (Maiolino et al. 2015): for two of them we set quite stringent 
constraints on the non-detection of the [CII] line, whereas the third one showed a detection from a region that is not centered on the galaxy. None of the $z \sim 7$ galaxies are detected in the far-IR continuum, which suggests a low dust mass. The only exception is A1689-zD1 at $z \sim 7.5$ with a clear detection of thermal dust emission (Watson et al. 2015). The above results suggest a change in galaxies' physical properties between $z \sim 6$ and $z \sim 7$.

In this work we present new observations of [CII] in four LBGs at $z \sim 7$. Throughout the paper, we adopt a cosmology with $\Omega_{\Lambda}=0.7, \Omega_{M}=0.3$ and $H_{0}=70 \mathrm{~km} / \mathrm{s} / \mathrm{Mpc}$. Magnitudes are in the $\mathrm{AB}$ system.

\section{SAMPLE SELECTION AND OBSERVATIONS}

We have recently completed CANDELSz7, an ESO spectroscopic large program using FORS2 at the VLT. Our goal is to systematically study the $\operatorname{Ly} \alpha$ emission in galaxies from $\mathrm{z}=5.5$ to $\mathrm{z}=7.2$ selected in the CANDELS fields (Grogin et al. 2011, Koekemoer et al. 2011). With the addition of previous data we have assembled a sample of $>120$ LBGs at $z \sim 7$ with homogeneous selection and deep spectroscopic observations (Pentericci et al. in preparation). In about $20 \%$ we detect the Ly $\alpha$ line, with EW in some cases as low as $5 \AA$. From this sample we have selected the seven brightest galaxies with: (1) precise redshifts between 6.6 and 7.2 from Ly $\alpha$ emission; (2) $S F R>15-30 M_{\odot} y r^{-1}$ based on UV emission, assuming the Kennicutt (1998) calibration with no dust correction. While these LBGs all have Ly $\alpha$ emission, the majority of them would not be selected by the usual LAE criteria because the lines only have modest EW.

During Cycle-3 we obtained observations for four of the seven galaxies approved in program 2015.1.01105.S. In Table 1 we show their optical properties. NTTDF6345 was part of one of our early studies (Pentericci et al. 2011), while the other galaxies were confirmed by CANDELSz7. COSMOS13679 has been independently confirmed by Stark et al. (2016). In Table 1 the coordinates reported are the HST Hband centroids in all cases except for NTTDF6345, which was detected with HAWK-I in Y-band (Castellano et al. 2010). For NTTDF6345 we had previously obtained lower S/N data in Cycle-2 (prog. 2013.1.01031.S ).

Objects were observed with ALMA in band 6 which has baselines between 14.7 and 376.9 meters, and provides a minimal resolution of 0.9 ". The center of the band was set at the redshift corresponding to the peak of the Ly $\alpha$ emission. Sensitivity was set to reach $\sim \log (\mathrm{L}[\mathrm{CII}])=7.5\left(\right.$ with $\mathrm{L}[\mathrm{CII}]$ in $\left.L_{\odot}\right)$. Observations were performed in Frequency Division Mode. Out of the four spectral windows, SPW1 was centred on the expected frequency of the [CII] line in the Upper Side Band. This spectral band was set to a spectral resolution of $10 \mathrm{~km}$ $\mathrm{s}^{-1}$. SPW0 was located on the continuum next to SPW1 (on the higher frequency side), while SPW2 and SPW3 were located in the Lower Side Band to sample the continuum.

The ALMA observations were carried out from April 2015 to March 2016. The number of antennas ranged from 36 to 46. The precipitable water vapour during the observations ranged between 0.82 and $3.0 \mathrm{~mm}$. The phases were centred at the positions reported in Table 1. The data were reduced with the Common Astronomy Software Application (CASA) and the final images were produced using the CLEAN task. The continuum image of each target was extracted using all the line-free channels of the four spectral windows, while spectral cubes were generated from the SPW1 dataset.
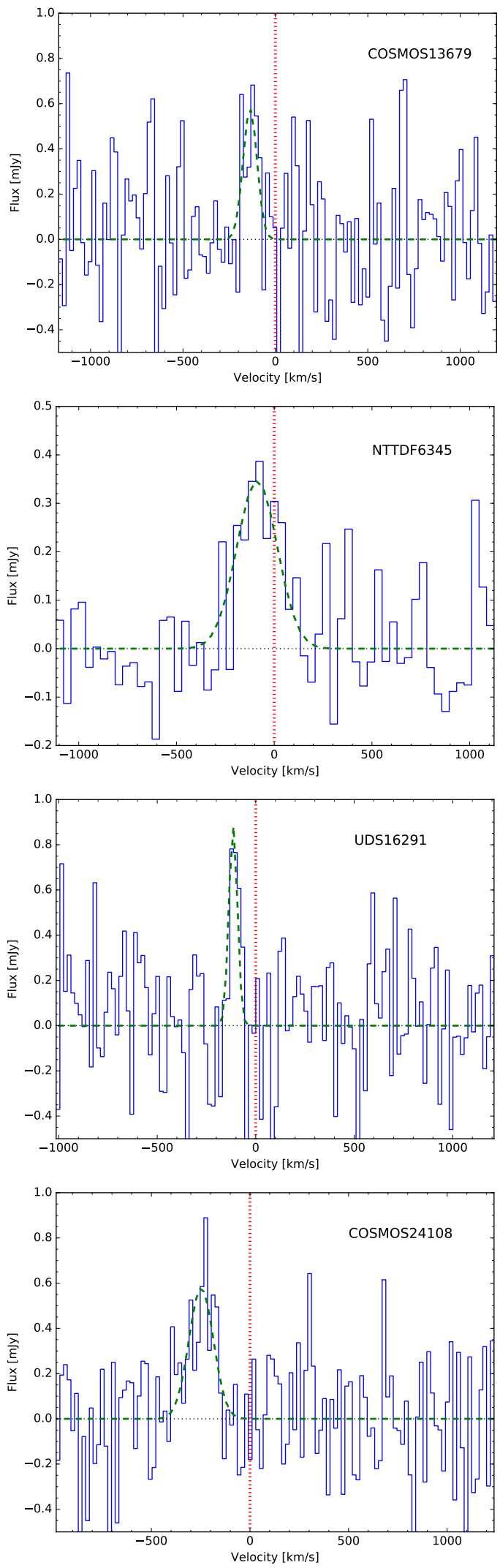

Figure 1. S. Spectra of the four galaxies in the region of the [CII] emission line. The velocities are shown with respect to that inferred from the Ly $\alpha$ which is marked with the dashed vertical lines. 
Table 1

Galaxies optical and spectroscopic properties

\begin{tabular}{lcccccccc}
\hline ID & RA & Dec & redshift & $\begin{array}{c}\text { SFR } \\
M_{\odot} y r^{-1}\end{array}$ & $\begin{array}{c}\text { Ly } \alpha \text { EW } \\
\AA\end{array}$ & $M_{U V}$ & $\begin{array}{c}M_{S E D} \\
10^{9} M_{\odot}\end{array}$ & ref \\
\hline COSMOS13679 & 150.0990366 & 2.3436268 & 7.1453 & 23.9 & 15 & -21.46 & 3.0 & 1,2 \\
NTTDF6345 & 181.4039006 & -7.7561900 & 6.701 & 25.0 & 15 & -21.57 & - & 3 \\
UDS16291 & 34.3561430 & -5.1856263 & 6.6381 & 15.8 & 6 & -20.97 & 0.6 & 1 \\
COSMOS24108 & 150.1972224 & 2.4786508 & 6.6294 & 29 & 27 & -21.67 & 3.9 & 1
\end{tabular}

Note. - Redshift reference: 1 Pentericci et al. in preparation; 2 Stark et al. (2016); 3 Pentericci et al. (2011)

\section{RESULTS}

\section{1. [CII] line detections}

In Table 2 we present our results: a [CII] emission line is detected for the three sources observed only in Cycle-3, with a $\mathrm{S} / \mathrm{N}=4.5$. For NTTDF6345 an emission line is observed separately in the Cycle- 2 and Cycle- 3 data-sets with a $\mathrm{S} / \mathrm{N}$ of 4.1 and 5.6 respectively, and a $\mathrm{S} / \mathrm{N}>6$ is obtained in the combination. Although the $\mathrm{S} / \mathrm{N}$ is modest, the spatial coincidence (or very close spatial association) between the [CII] and the near-IR counterparts, and the consistent small shift with respect to the redshift determined from the Ly $\alpha$ emission all argue for the reality of the detections. In addition in three out of four cases the detections are spatially resolved. In Figure 1 for each source we present the spectrum of the $[\mathrm{CII}]$ region with a rebinning of $40 \mathrm{~km} \mathrm{~s}^{-1}$ for NTTDF6345 and $20 \mathrm{~km} \mathrm{~s}^{-1}$ for the other sources: the vertical dotted line at $\mathrm{v}=0 \mathrm{~km} \mathrm{~s}^{-1}$ indicates the redshift determined from the Ly $\alpha$ emission. The maps of the line emission are shown in Figure 2, extracted with a spectral width of $440 \mathrm{~km} \mathrm{~s}^{-1}$ for NTTDF6345, $280 \mathrm{~km}$ $\mathrm{s}^{-1}$ for COSMOS24108, $100 \mathrm{~km} \mathrm{~s}^{-1}$ for UDS16291 and 120 $\mathrm{km} \mathrm{s}^{-1}$ for COSMOS13679. The black crosses indicates the centroid of the Y-band image for NTTDF6345 and of the HST $\mathrm{H}$-band images for the other galaxies.

Thermal far-infrared continuum is not detected in any of the galaxies: the limits on the total IR-luminosity convert into limits on the dust obscured $S F R_{\text {dust }}$ that are reported in Table 2 (assuming a Kennicutt (1998) relation with a Salpeter initial mass function).

\subsection{Offsets between [CII] and rest-frame UV position}

In Table 2 we report the offset between the near-IR coordinates and the ALMA detections. In two cases the shifts are consistent with the ALMA astrometric uncertainty (0.1$0.15^{\prime \prime}$ ), while for COSMOS24108 and COSMOS13679 they are larger. Dunlop et al. (2016) recently noted that the HST and ALMA astrometry of the HUDF field presented both a systematic shift of 0.25 " to the south and a random shift of up to $0.5^{\prime \prime}$. Spatial offsets of up to $0.5^{\prime \prime}$ are clearly evident in most of the LBGs observed by Capak et al. (2015) and for the $z \sim 6$ galaxy WMH5 observed by Willot et al. (2015) $\left(\sim 0.4^{\prime \prime}\right)$, while in the other galaxy CLM1, the [CII] emission is co-spatial with the UV continuum.

To further investigate this issue we looked at bright serendipitous sources detected in the continuum band in our fields. We find at least one source per field and we measure a shift between $0.1^{\prime \prime}$ and $0.6^{\prime \prime}$ in random directions between the HST (or HAWK-I) counterpart and the ALMA detection. Given the depth of the CANDELS images $(H \sim 27$ at $5 \sigma)$ it is unlikely that the ALMA detections are associated to other objects undetected in the H-band. Note that for NTTDF6345 where we only have HAWK-I images, the shift is negligible both for the serendipitous source and the LBG. We conclude that there are still substantial uncertainties in the relative ALMA-HST astrometry. In summary, for UDS16291 and NTTDF6345 the [CII] detections are centered at the position of the near-IR sources, and for COSMOS13679 the offset $(0.4$ ") is within the range of those reported in the literature and measured for the serendipitous sources: for these three galaxies we conclude that the [CII] emission comes from the same region as the bulk of the SF observed in the near-IR images. For COSMOS24108 the offset is slightly larger than the range reported in the literature, and while the [CII] emission is almost certainly associated to the source, it could actually come from an external region of the galaxy, not coincident with the bulk of the star formation. A similar case was already observed in BDF3299 at $z=7.109$ (Maiolino et al. 2015): for this galaxy we concluded that the $[\mathrm{CII}]$ emission arises from an external accreting/satellite clump of neutral gas, in agreement with recent models of galaxy formation (Vallini et al. 2015). While the identification with another transition from a foreground galaxy, which by chance happens to be at $0.8^{\prime \prime}$ from COSMOS24108, is unlikely, we cannot completely discard the possibility of a spurious detection given the low $\mathrm{S} / \mathrm{N}$.

\section{DISCUSSION}

\section{1. [CII] - Ly $\alpha$ velocity shifts}

The [CII] line traces the systemic redshift of the sources, unlike the Ly $\alpha$ line which is typically red-shifted by up to several hundreds $\mathrm{km} \mathrm{s}^{-1}$ (Erb et al. 2014, Trainor et al. 2016) consistent with the presence of outflowing gas, although the final observed Ly $\alpha$ profiles depend on many factors such as geometry, gas covering fraction, dust and IGM ionization state. For our galaxies the velocity shifts are not very large, of the order of $100-200 \mathrm{~km} \mathrm{~s}^{-1}$, smaller than those reported at $z \sim 3$ for galaxies with similar UV luminosities. Specifically Erb et al. (2014) measure shifts of up to $1000 \mathrm{~km} \mathrm{~s}^{-1}$ and average values of $400 \mathrm{~km} \mathrm{~s}^{-1}$ for LBGs with $M_{U V}<-21$. The mean shift is also lower than those reported by Willott et al. (2015), 430 and $275 \mathrm{~km} \mathrm{~s}^{-1}$ respectively for their two $z \sim 6$ galaxies 11 The small shifts in our galaxies are particularly significant given that in general objects with low Ly $\alpha$ emission have larger velocity offsets. A similar tentative evidence for smaller Ly $\alpha$ velocities at $z \sim 7$ compared to $z \sim 3$ was recently reported by Stark et al. (2015) using the UV nebular CIII] $\lambda 1909$ doublet to determine the systemic redshift in two distant LBGs.

The velocity of $\operatorname{Ly} \alpha$ compared to the systemic redshift is very relevant when interpreting the line visibility during the reionization epoch, in the presence of a partly neutral IGM

${ }^{11}$ For the Capak et al. (2015) sources this comparison is not possible since their UV redshifts are not from Ly $\alpha$ emission 
Table 2

Galaxies ALMA properties

\begin{tabular}{ccccccccccc}
\hline ID & $\begin{array}{c}\left.\lambda_{0}[\mathrm{CII}]\right) \\
\mathrm{mm}\end{array}$ & $\begin{array}{c}{[\mathrm{CII}] \mathrm{flux}} \\
\mathrm{Jy} \mathrm{km} / \mathrm{s}\end{array}$ & $\begin{array}{c}\text { FWHM [CII] } \\
\mathrm{km} / \mathrm{s}\end{array}$ & $\mathrm{S} / \mathrm{N}$ & $\begin{array}{c}r_{m s_{\text {cont }}} \\
\mu J_{y b e a m}{ }^{-1}\end{array}$ & $\begin{array}{c}t_{\text {int }} \\
\mathrm{s}\end{array}$ & $\begin{array}{c}\Delta \text { pos } \\
\operatorname{arcsec}\end{array}$ & $\begin{array}{c}\Delta v \\
\mathrm{~km} \mathrm{~s}^{-1}\end{array}$ & $\begin{array}{c}M_{\text {dyn }} * \sin (i)^{2} \\
10^{9} M_{\odot}\end{array}$ & $\begin{array}{c}S F R_{\text {dust }} \\
M_{\odot} y r^{-1}\end{array}$ \\
\hline COSMOS13679 & $1.28426 \pm 0.00006$ & $5.9 \times 10^{-2}$ & $90 \pm 35$ & 4.5 & 14 & 2782 & 0.4 & 135 & $<2.5$ & $<6.2$ \\
NTTDF6345 & $1.2143 \pm 0.0002$ & $1.6 \times 10^{-1}$ & $250 \pm 70$ & 6.1 & 16 & 2087 & 0.0 & 110 & $<18$ & $<5.7$ \\
UDS16291 & $1.20438 \pm 0.00003$ & $6.3 \times 10^{-2}$ & $50 \pm 15$ & 4.5 & 20 & 2117 & 0.1 & 110 & $<0.7$ & $<6.6$ \\
COSMOS24108 & $1.20249 \pm 0.00007$ & $9.2 \times 10^{-2}$ & $150 \pm 40$ & 4.5 & 18 & 2177 & 0.8 & 240 & $<6.5$ & $<6.2$
\end{tabular}
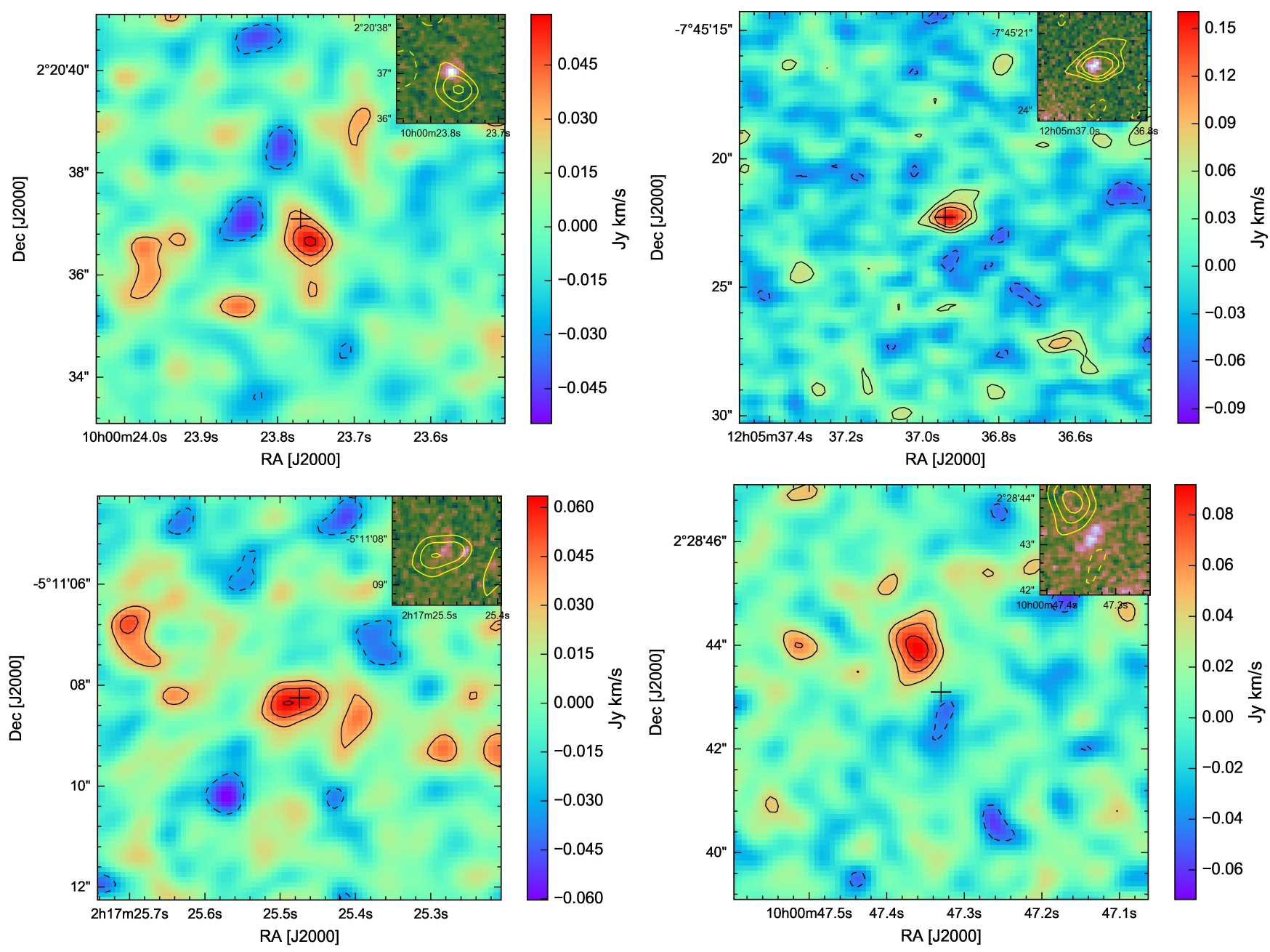

Figure 2. Maps of the [CII] emission for COSMOS13679 (upper-left), NTTDF6345 (upper-right), UDS16291(lower-left) and COSMOS24108 (lower-right). Contours are $-2,2,3,4 \sigma$ level. The crosses indicate the position of the near-IR detections. In the insets we show the near-IR images with ALMA contours overlayed.

(Dijkstra et al.2011). Smaller velocity offsets imply that Ly $\alpha$ is closer to resonance and more easily quenched by a neutral IGM. If at redshift 6 and 7 the offsets are as large as those found for lower redshift LBGs, the IGM must be very neutral to produce the drop in $\operatorname{Ly} \alpha$ fraction that is observed between these two epochs (Pentericci et al. 2014). On the other hand, if the velocity offsets were much smaller, as our observations indicate, the drop in the $\operatorname{Ly} \alpha$ visibility could be produced by an IGM that is still substantially more ionized (Mesinger et al. 2015).

\subsection{SFR-L[CII] relation}

In Figure 3 we show the SFR-L[CII] relation for our galaxies and previously observed sources. We plot COSMOS24108 with a different symbol because it is not certain whether its [CII] emission is from the main galaxy or just from a clump in its outskirt, in which case the point would shift $\sim 1$ dex to the left. We remark that the SFR for our sources as well as previous $z \sim 7$ ones are UV-based, with no correction for dust extinction. As stated above the upper limits on the $S F R_{d u s t}$ are very low, at least for our galaxies. The SFR for the Capak et al. (2015) sample include both the UV and dust obscured contribution. Finally for the Willot et al. sample we plot SEDderived SFR. Our galaxies are a factor of 2-3 less luminous in 
$\mathrm{L}[\mathrm{CII}]$ than $z \sim 5.5$ galaxies with similar SFRs. They also fall below the SFR-L[CII] relation of low redshift star forming galaxies and low metallicity galaxies (black solid and dashed lines De Looze et al. 2014).

Previous observations of $z \sim 7$ galaxies failed to detect the [CII] emission. Some of the galaxies were fainter than ours, but few others were in the same range and in these cases the limits reached were deep enough to detect [CII] if the emission was at the same level as in our sources (Maiolino et al. 2015, Schaerer et al. 2015).

However we note that previously observed sources were either selected as Ly $\alpha$ emitters or were LBGs but their spectra showed a Ly $\alpha$ emission with very high EW (typically $>40 \AA$ ). The only exception z8-GND-5296 at $\mathrm{z}=7.5$ which has modest Ly $\alpha$ emission, but in this case the L[CII] limit is very shallow (Schaerer et al. 2015). Our four new sources have Ly $\alpha$ emission with low EW (Table 1). The Ly $\alpha$ emission strength is known to depend on the presence on dust and possibly metallicity (Raiter et al. 2010): although the derivation of metallicity is not easy, several studies indicate that LAEs are more metal poor galaxies compared to the rest of the LBG population (e.g. Song et al. 2014). Metallicity plays an important role in shaping the SFR-L[CII] relation: in Figure 3 we show different metallicity dependent relations produced by a recent study of Vallini et al. (2015) based on high-resolution, radiative transfer cosmological simulations. The different lines have $\mathrm{Z}=0.05,0.1$ and $0.2 \mathrm{Z}_{\odot}$ respectively, and for metallicity $\leq 0.1$ are consistently below the relation found for local galaxies. Therefore the contradictory results for $z \sim 7$ galaxies might be due to different intrinsic metallicity.

Alternatively galaxies could be caught in different evolutionary stages: it has been suggested that molecular clouds in the central parts of primordial galaxies could be rapidly disrupted by stellar feedback hence suppressing the emission of [CII]. Clumps of neutral gas (or small satellites) in the outer regions of such galaxies, could survive photo-ionization and still show [CII] emission, as a consequence of the diffuse farUV radiation emitted by the primary galaxy. This could be the case of COSMOS24108, where the [CII] emission is offset from the near-IR source, and could come from one such clump. A similar occurrence was also observed in BDF3299 at $\mathrm{z}=7.109$ where the $[\mathrm{CII}]$ emission was displaced by the near-IR source by $4 \mathrm{kpc}$. We refer to Maiolino et al. (2015) for an extensive discussion of this scenario.

\subsection{Dynamical masses}

Assuming the sources have ordered motions, we can estimate the dynamical masses based on the [CII] velocity dispersion. We follow the method described in Wang et al. (2013) who approximate $M_{d y n}=1.16 \times 10^{5} V_{\text {cir }}^{2} D$, where $V_{\text {cir }}$ is the circular velocity in $\mathrm{km} \mathrm{s}^{-1}, \mathrm{D}$ is the size in $\mathrm{kpc}, V_{\text {cir }}=$ $1.763 \sigma[C I I] / \sin (i)$, and $i$, the disk inclination angle. Our sources are only marginally resolved so we assume an upper limit on D from the resolution of $0.9^{\prime \prime}$. We obtain masses between $0.7-18 \times 10^{9} M_{\odot}$. We can compare these values to the total stellar masses determined by a classical SED fitting of the multi-wavelength photometry for the 3 CANDELS sources. We use the photometry from the CANDELS catalogs, including deep IRAC data essential for sampling the rest-frame optical emission at these redshifts. We include the contribution from nebular emission lines, which can strongly contaminate the IRAC bands. The stellar masses are reported in Table 1 and are very similar to the dynamical masses, espe-

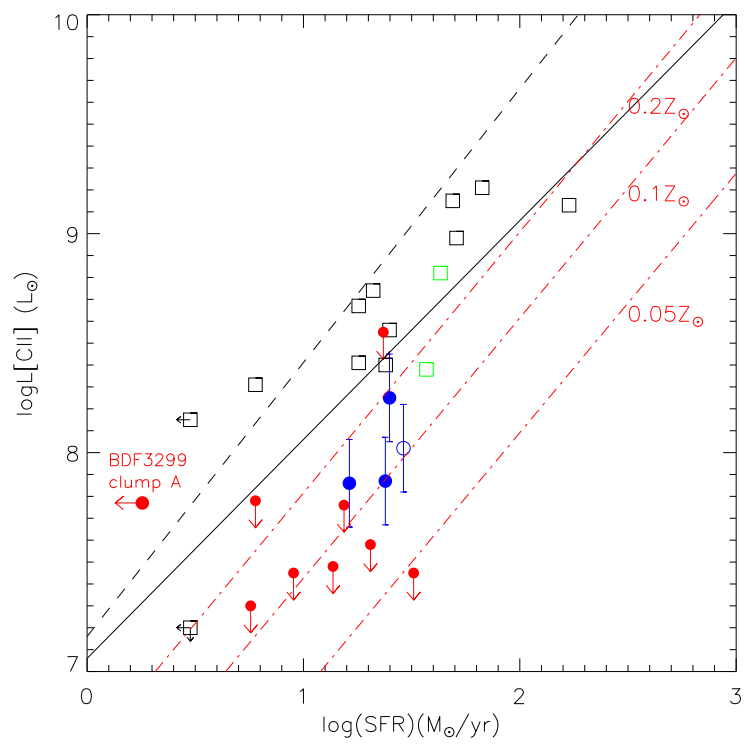

Figure 3. S. The blue circles represent the galaxies of the present study with the empty circle indicating COSMOS24108 for which the association between the $[\mathrm{CII}]$ emission and the optical galaxy is uncertain. Red circles are previous $z \sim 7$ objects (Schaerer et al. 2014; Maiolino et al. 2015). Empty black squares are $\mathrm{Z} \sim 5.5$ galaxies from Capak et al. (2015); green squares are $\mathrm{z} \sim 6$ objects from Willott et al. (2015). The black lines show the relations for local star forming galaxies and star-bursts (not-including ULIRGs) (solid) and low redshift metal poor dwarf galaxies (dashed) (De Looze et al. 2014). The red dot-dashed lines are the resulting relations from simulations (Vallini et al. 2015) for $Z=0.05 Z_{\odot}, 0.1 Z_{\odot}$ and $0.2 Z_{\odot}$ respectively.

cially considering the uncertainty due to the unknown inclination angle $i$.

\section{CONCLUSIONS}

We have presented ALMA [CII] emission line detections in four $z \sim 7$ galaxies. Our observations demonstrate that it is possible to detect [CII] during the reionization epoch and that the line luminosity is $\sim 3$ times lower than expected on the basis of the lower redshift relations. We find evidence for reduced velocity offsets of the $\operatorname{Ly} \alpha$ emission compared to $z \sim 3$ LBGs: if confirmed this could have important implications for reionization models, since small shifts alleviate the need for a very neutral IGM to reproduce the observed decline of Ly $\alpha$ emission at $z \sim 7$. These results can help us tune future ALMA observations of high-z LBGs where no Ly $\alpha$ emission is visible but where precise photometric redshifts exist, as in the CANDELS fields. Given that in the reionization epoch most galaxies do not show Ly $\alpha$ emission, this means that [CII] can be an efficient alternative to derive the redshift for the majority of the galaxy population. While larger samples of targets with different luminosities and Ly $\alpha$ properties are needed to put our conclusions on firmer grounds, additional diagnostic can be used to interpret the physical conditions in these objects. For example in case of photo-ionization feedback [NII] at $205 \mu \mathrm{m}$ can be much stronger than [CII] (Pavesi et al. 2016). Alternatively the [OII] line at $88 \mathrm{~m} \mu$ could be brighter than [CII] in chemically unevolved systems (Cormier et al. 2015 ) as recently observed in a dust-poor galaxy at $z=7.2$ (Inoue et al. 2016).

\section{REFERENCES}

Capak, P. L., Carilli, C., Jones, G., et al. 2015, Nature, 522, 455 Castellano, M., Fontana, A., Paris, D., et al. 2010, A\&A, 524, A28 
Cormier, D., Madden, S. C., Lebouteiller, V., et al. 2015, A\&A, 578, A53 De Looze, I., Cormier, D., Lebouteiller, V., et al. 2014, A\&A, 568, A62 Dijkstra, M., Mesinger, A., \& Wyithe, J. S. B. 2011, MNRAS, 414, 2139 Dunlop, J. S., McLure, R. J., Biggs, A. D., et al. 2016, arXiv:1606.00227 Erb, D. K., Steidel, C. C., Shapley, A. E., Pettini, M., \& Adelberger, K. L. 2004, ApJ, 612, 122

Finkelstein, S. L., Ryan, R. E., Jr., Papovich, C., et al. 2015, ApJ, 810, 71

Finkelstein, S. L., Papovich, C., Dickinson, M., et al. 2013, Nature, 502, 524

Giallongo, E., Grazian, A., Fiore, F., et al. 2015, A\&A, 578, A83

Grogin, N. A., Kocevski, D. D., Faber, S. M., et al. 2011, ApJS, 197, 35

Inoue, A. K., Tamura, Y., Matsuo, H., et al. 2016, Science, 352, 1559

Knudsen, K. K., Richard, J., Kneib, J.-P., et al. 2016, MNRAS, 462, L6

Koekemoer, A. M., Faber, S. M., Ferguson, H. C., et al. 2011, ApJS, 197, 36

Maiolino, R., Carniani, S., Fontana, A., et al. 2015, MNRAS, 452, 54

Mesinger, A., Aykutalp, A., Vanzella, E., et al. 2015, MNRAS, 446, 566

Oesch, P. A., van Dokkum, P. G., Illingworth, G. D., et al. 2015, ApJ, 804, L30

Ota, K., Walter, F., Ohta, K., et al. 2014, ApJ, 792, 34

Pavesi, R., Riechers, D. A., Capak, P. L., et al. 2016, arXiv:1607.02520

Pentericci, L., Vanzella, E., Fontana, A., et al. 2014, ApJ, 793, 113
Pentericci, L., Fontana, A., Vanzella, E., et al. 2011, ApJ, 743, 132 Raiter, A., Schaerer, D., \& Fosbury, R. A. E. 2010, A\&A, 523, A64 Robertson, B. E., Ellis, R. S., Furlanetto, S. R., \& Dunlop, J. S. 2015, ApJ, 802, L19

Schaerer, D., Boone, F., Zamojski, M., et al. 2015, A\&A, 574, A19 Schenker, M. A., Stark, D. P., Ellis, R. S., et al. 2012, ApJ, 744, 179 Song, M., Finkelstein, S. L., Gebhardt, K., et al. 2014, ApJ, 791, 3 Stark, D. P., Walth, G., Charlot, S., et al. 2015, MNRAS, 454, 1393 Stark, D. P., Ellis, R. S., Charlot, S., et al. 2016, arXiv:1606.01304

Trainor, R. F., Steidel, C. C., Strom, A. L., \& Rudie, G. C. 2015, ApJ, 809, 89

Vallini, L., Gallerani, S., Ferrara, A., Pallottini, A., \& Yue, B. 2015, ApJ, 813,36

Wang, R., Wagg, J., Carilli, C. L., et al. 2013, ApJ, 773, 44

Watson, D., Christensen, L., Knudsen, K. K., et al. 2015, Nature, 519, 327

Willott, C. J., Carilli, C. L., Wagg, J., \& Wang, R. 2015, ApJ, 807, 180

Zitrin, A., Labbé, I., Belli, S., et al. 2015, ApJ, 810, L12

This paper makes use of the following ALMA data: ADS/JAO.ALMA\#2015.1.01105.S. ALMA is a partnership of ESO (representing its member states), NSF (USA) and NINS (Japan), together with NRC (Canada), NSC and ASIAA (Taiwan), and KASI (Republic of Korea), in cooperation with the Republic of Chile. The Joint ALMA Observatory is operated by ESO, AUI/NRAO and NAOJ.

This paper is based on data obtained with ESO program 190.A-0685.

SC and RM acknowledge financial support from the Science and Technology Facilities Council (STFC) 